\title{
Maintenance of demographic and hematological profiles in a long-lasting dengue fever outbreak: implications for management
}

Andréia Moreira dos Santos Carmo ${ }^{1,2}$, Rodrigo Buzinaro Suzuki ${ }^{1,3}$, Michele Marcondes Riquena?', André Eterovic $^{4}$ and Márcia Aparecida Sperança ${ }^{1 *}$

\begin{abstract}
Background: Dengue fever (DF) outbreaks present regionally specific epidemiological and clinical characteristics. In certain medium-sized cities (100 000-250 000 inhabitants) of São Paulo State, Brazil, and after reaching an incidence of 150 cases/100 000 inhabitants ("epidemiological threshold"), clinical diagnosis indicated dengue virus (DENV) infection. During this period, other seasonally infectious diseases with symptoms and physical signs mimicking DF can simultaneously occur, with the consequential overcrowding of health care facilities as the principal drawbacks. Confirmation of clinical diagnosis of DF with serological tests may help in avoiding faulty diagnosis in patients, who might later undergo dengue hemorrhagic fever (DHF) and the dengue-shock syndrome (DSS). Furthermore, demographic and hematological profiles of patients are useful in detecting specific early characteristics associated to DF, DHF and DSS.
\end{abstract}

Methods: From March to June, 2007, 456 patients from Marilia in northwest São Paulo State who had only been diagnosed for DF by clinical criteria, underwent serologic testing for non-structural 1 (NS1) DENV antigens. Individual results were used in comparative analysis according to demographic (gender, age) and hematological (leukocyte and platelet counts, percentage of atypical lymphocytes) profiles. Temporal patterns were evaluated by subdividing data according to time of initial attendance, using recorded variables as predictors of DENV infection in logistic regression models and ROC curves.

Results: Serologic DENV detection was positive in $70.6 \%$ of the patients. Lower leukocyte and platelet counts were the most important factors in predicting DENV infection (respective medians DENV $+=3715 \mathrm{cell} / \mathrm{sl}$ and DENV- $=6760 \mathrm{cells} / \mathrm{ml}$, and DENV $+=134896 \mathrm{cell} / \mathrm{ml}$ and DENV- = $223872 \mathrm{cells} / \mathrm{ml}$ ). Furthermore, all demographic and hematological profiles presented a conservative temporal pattern throughout this long-lasting outbreak.

Conclusions: As consistency throughout the epidemic facilitated defining the conservation pattern throughout the early stages, this was useful for improving management during the remaining period.

Keywords: Clinical diagnosis, Demographic profile, Dengue fever, Dengue- outbreak pattern, Epidemiological threshold, Hematological profile

\footnotetext{
* Correspondence: marcia.speranca@ufabc.edu.br

${ }^{1}$ Center for Natural and Human Sciences, Universidade Federal do ABC,

Campus São Bernardo do Campo, 09606-070 São Bernardo Do Campo, São

Paulo, Brasil

Full list of author information is available at the end of the article
} 


\section{Multilingual abstracts}

Please see Additional file 1 for translations of the abstract into the five official working languages of the United Nations.

\section{Background}

The dengue virus of the genus Flavivirus (family Flaviviridae) is represented by four serotypes (DENV1 to 4), which are transmitted to humans by infected female Aedes aegypti and A. albopictus mosquitoes, widely distributed throughout tropical and subtropical regions [1]. According to the World Health Organization [2], $40 \%$ of the population world-wide run the risk of DENV infection, with an estimated 50 to 100 million cases annually and around 20000 fatalities.

Disease manifestation in DENV infected individuals ranges from absence or unspecified to classical dengue fever (DF), characterized by fever associated to muscle, joint and retro-orbital pain, photophobia and red bodyrash. The most severe and lethal forms of the disease are characterized by increased vascular permeability, plasma leakage, thrombocytopenia and hemorrhagic manifestations [3, 4]. Risk factors, which vary in populations world-wide [5], include sequential DENV infection with a different virus serotype, diversity in genetic characteristics of both virus and host, vector dispersion and adaptability, and sequential virus serotype infection.

In medium-sized cities (100 000-300 000 inhabitants), DF outbreaks typically last for four to six months during the hot and rainy seasons, with specific epidemiological and clinical characteristics in space and time [6, 7]. In the New World, the spread of DENV has increased fivefold over the latter decades, with outbreaks occurring every three to five years through co-circulation of the four serotypes of DENV, with total severe dengue case fatality reaching $1.2 \%$ [8]. Within this scenario and since 2000, Brazil has accounted for more than $70 \%$ of DENV cases in the Americas, with a period of outstanding A. aegypti dispersion [9]. By mid-2007 and in all the dengue epidemics, adults from urban and surrounding locations were the most affected. However, in some regions and from the second half of 2007, there has been a change in this pattern, with a significant increase in severe dengue hemorrhagic cases in under15-year-old children in the northeast and Rio de Janeiro State [10]. Nonetheless, the adult population continues to be the most affected. Hence, there is the need for regional studies to facilitate early recognition of any modification in the DENV epidemiological pattern, to thus improve control and treatment procedures.

In São Paulo State, concentration of DENV cases in urban centers has changed, with an increased incidence in small and medium-sized towns. In 2007, $52 \%$ of the cases were reported in towns with less than 100000 inhabitants, with $16 \%$ occurrence in counties with populations ranging between 100000 and 500000 (Ministry of Health, 2007).

Marilia, a town in the west of São Paulo State, $450 \mathrm{~km}$ from the capital, with 216745 inhabitants (IBGE 2010) and a large population flow due to economic relevance, represents a model for investigating DENV epidemiology in medium-sized towns state-wide. In the 2007 Mariliaoutbreak, which reached 550 cases/100 000 inhabitants, in the first instance, only around 300 cases were confirmed through serological analysis. Subsequently, all suspected cases were required to be notified and submitted to hematological check-ups for detection and posterior treatment of severe hemorrhagic cases, whence the consequential overcrowding of health facilities.

During outbreaks, the use of such "epidemiological threshold" procedures in accordance with clinical criteria for DENV diagnosis, but without serological confirmation, imposes another significant problem; the co-occurrence of various seasonal infectious diseases with similar symptoms. These include other arboviruses, such as the flavivirus Saint Louis Encephalitis [11], Chikungunya fever [12, 13], Zika fever [14], leptospirosis [15, 16], visceral leishmaniasis [17], and malaria [18].

Certain demographic characteristics, such as the age of patients diagnosed with DENV, can be associated to distinct epidemiological patterns of DENV transmission [19]. Furthermore, early identification of the hematological profile during the fever period can differentiate DENV from other infectious diseases [20]. Thus, the aim hereby was to assess the demographic and hematological profiles of patients under treatment at the Marilia Hemocenter during the 2007 DF outbreak, and diagnosed by clinical criteria according to DF epidemiological threshold procedures. DENV infection in such individuals was investigated a posteriori (retrospectively) by way of serological testing.

\section{Methods}

Patients and laboratory analysis

When, during the 2007 outbreak, serologically confirmed dengue incidence reached the epidemiological threshold of 150/100 000 inhabitants in Marilia, 754 patients were diagnosed for dengue only by clinical means. They were attended to at the Marilia Hemocenter from March, 7 to June, 4. From the onset, they underwent a posteriori investigation to assess the serological status (DENV presence/absence), and demographic (gender and age) and hematological (leukocyte and platelet counts, and percentage of atypical lymphocytes) profiles of each. Only individuals with complete records (gender, age, leukocyte and platelet counts, and percentage of atypical lymphocytes) came under analysis. Hematocrit results, also monitored from the beginning, showed less 
than $6 \%$ of investigated patients (26 out of 456) presented values above standard levels (42\% for under-12year-old children, $44 \%$ for adult women and $50 \%$ for adult men) [21]. Percentages of red blood cells among under-12-year-olds $(n=34)$ ranged from 27.5 to 45.5 (median - 38.3; first and third quartiles 35.3 and 41.3, respectively); among women ( $n=226)$ from 32.2 to 49.6 (median - 40.0; first and third quartiles 37.8 and 42.0, respectively); and among men $(n=196)$ from 29.1 to 49.9 (median - 44.1, first and third quartiles 41.5 and 45.8 respectively). However, since according to clinical and laboratory follow up, no patient presented hemoconcentration, these data were not included for further analysis. Blood-samples were collected in EDTA tubes from each patient for cell counting. The remaining material was centrifuged for plasma separation, and after frozen at $-20{ }^{\circ} \mathrm{C}$. Atypical lymphocytes were investigated by blood smear microscopic examination after Giemsa staining. Plasma samples underwent DENV serological diagnosis by enzyme linked immunosorbent assay detection of non-structural 1 (NS1) antigen, using the “Focus Diagnostics' Dengue NS1 Antigen DxSelect ${ }^{\mathrm{tx}}$ Assay", according to manufacturers' instructions. Research protocol was approved by the Ethics Committee on Human Research of the Marilia Medical School (number 069/03).

\section{Statistical analysis}

Among the 456 patients with complete records, and based on serological outcomes (positive cases $=\mathrm{DENV}+$; negative cases = DENV-) two groups were described through distribution by gender (labeled as SEX, with males coded as 1 , females as 0 ), age in years (AGE), leukocyte and platelet counts (respectively, LEU and PLA, both in $\log _{10}$ cells $/ \mathrm{ml}$ ), and percentage of atypical lymphocytes (AL\%). On the first day of attendance at the Hemocenter, DAY, counted from the start of the 89days-interval, was also used in the survey. Within each group, these variables were evaluated separately by descriptive statistics. The groups were compared using the $X^{2}$ test for SEX (in a $2 \times 2$ contingency table), and the Mann-Whitney for the remaining variables. Bivariate analysis was by Spearman rank correlations. In a contrary approach, the six variables were evaluated as independent explanatory factors, and according to their probability to predict DENV serological outcome. Logistic regression models were used to detect significant effects of each factor on the dichotomic response variable (DENV+ as 1, DENV- as 0). An additive model including all the six factors was also built. Parameters from the first set of models (explanatory factors used individually) described the relationship between each factor and the response variable by means of logit functions. Analysis was with the Pezzulo (2012) free online device for calculating logistic regression. Additional estimates of confidence intervals were obtained with Statistica 8.0 (Statsoft Co) software. Akaike Information Criteria (AIC values) were computed in an $\mathrm{R}$ software package. For all the variables except SEX, sigmoidal curves relating values to the response variable were plotted. Receiver operating characteristic (ROC) curves were plotted for all the factors except SEX. Confidence intervals for both specificity and sensibility were compiled with the $R$ package pROC [22], as was the case of comparison among areas below the curves (AUC).

We evaluated how the different subsets of patients obtained from distinct periods of first attendance at the Marilia Hemocenter could generate particular individual explanations of serological outcome with the selected factors. Data were divided into four subsets, each comprised of an equal number of individuals (s1 to s4; $N=114$ in all), with the first and the last quarter of patients to arrive at the Marilia Hemocenter being placed accordingly in the subsets. Each of the four was treated as an entire set of data to so build up new logistic regression and ROC curves. Comparison was between these subsets and the entire sample. Unless otherwise explicitly stated, $P$-values were considered following Bonferroni correction based on the number of similar tests.

\section{Results}

As an indication of the absence of sampling bias, data from the discarded group of patients with incomplete records $\left(\mathrm{N}_{\mathrm{out}}=330\right)$ did not differ significantly from those of the group of patients included in the analysis $\left(\mathrm{N}_{\mathrm{in}}=456\right)$, when considering (a) sex $(2 \times 2$ contingency table: $X^{2}=35.4 ; P=0.3775 ; \mathrm{N}_{\text {out }}=330$ ), (b) age (Mann-Whitney $\mathrm{U}=28$ 940; $P=0.1907 ; \quad \mathrm{N}_{\text {out }}=137$ ), (c) day of the first attendance at the Hemocenter (Mann-Whitney $\mathrm{U}=73$ 110; $P=0.4982 ; \quad \mathrm{N}_{\text {out }}=330$ ), or (d) representativeness of original location (number of patients in both groups from 23 Marilia districts compared by Spearman rank correlation: $\mathrm{rS}=0.826 ; P<0.0001$; $\mathrm{N}_{\text {out }}=82$ ).

A summary of variables and univariate comparison between the two groups of serological outcome appears in Table 1. Among the 456 patients evaluated, 322 (70.6\%) were serum positive (DENV+). In the latter, $48.1 \%$ were males with median age of 35 years. The median time of the first attendance at the Marilia Hemocenter since sampling started was 41 days. Among serumnegative patients (DENV-), males comprised $42.6 \%$ with a median age of 29 years. The Median time from first attendance was 38.5 days. For the three variables SEX, AGE, and DAY, differences among groups were insignificant. Groups differed significantly as regards hematological conditions. Leukocyte and platelet counts 
Table 1 Demographic and hematological status of DENV+ and DENV- patients in the 2007 Marilia outbreak

\begin{tabular}{|c|c|c|c|c|c|}
\hline \multicolumn{2}{|c|}{ Variable } & \multirow{2}{*}{$\frac{\text { DENV+ }}{48.1}$} & \multirow{2}{*}{$\begin{array}{l}\text { DENV- } \\
42.5\end{array}$} & \multirow{2}{*}{$\begin{array}{l}\text { Test } \\
1.19\end{array}$} & \multirow{2}{*}{$\frac{P}{0.2748}$} \\
\hline SEX & $\%$ males & & & & \\
\hline \multirow[t]{4}{*}{ DAY } & Mean & 42.25 & 37.84 & 19,050 & 0.0485 \\
\hline & Standard deviation & 19.906 & 20.396 & & \\
\hline & Median & 41 & 38.5 & & \\
\hline & Range & $(0-86)$ & $(0-86)$ & & \\
\hline \multirow[t]{4}{*}{ AGE } & Mean & 37.08 & 32.55 & 18,410 & 0.0137 \\
\hline & Standard deviation & 18.558 & 19.774 & & \\
\hline & Median & 35 & 29 & & \\
\hline & Range & $(0-85)$ & $(1-78)$ & & \\
\hline \multirow[t]{4}{*}{ LEU } & Mean & 3.57 & 3.83 & 7654 & $<0.0001$ \\
\hline & Standard deviation & 0.205 & 0.197 & & \\
\hline & Median & 3.56 & 3.83 & & \\
\hline & Range & $(2.95-4.30)$ & (3.28-4.39) & & \\
\hline \multirow[t]{4}{*}{ PLA } & Mean & 5.12 & 5.33 & 8830 & $<0.0001$ \\
\hline & Standard deviation & 0.214 & 0.180 & & \\
\hline & Median & 5.13 & 5.35 & & \\
\hline & Range & $(4.00-5.69)$ & $(4.64-5.87)$ & & \\
\hline \multirow[t]{4}{*}{$\mathrm{AL} \%$} & Mean & 6.52 & 2.50 & 13,160 & $<0.0001$ \\
\hline & Standard deviation & 6.791 & 3545 & & \\
\hline & Median & 4 & 1 & & \\
\hline & Range & $(0-39)$ & $(0-20)$ & & \\
\hline
\end{tabular}

Demographic and hematological status of patients from two groups based on serological outcomes in Dengue virus testing (DENV+ $N=322$, DENV- $N=134)$. Gender (SEX), age (AGE, in years), leukocyte and platelet counts (respectively LEU and PLA, both in $\log _{10}$ cells $/ \mathrm{ml}$ ), percentage of atypical lymphocytes (AL\%) and day of the first attendance at the Marilia Hemocenter (DAY, counted from March 07, 2007, when sampling started) were recorded for 456 patients. All test values are Mann-Whitney U comparisons among groups, except for SEX $\left(X^{2}\right.$ applied to a two-way contingency table). $P$-values lower than critical $\left(P_{c}=0.05 / 6=0.0083\right.$, considering six contrasts in a Bonferroni correction) were shown in bold

were lower in positive patients (leukocyte medians: $\mathrm{DENV}+=3715$ cells $/ \mathrm{ml}$ and DENV-=6760 cells $/ \mathrm{ml}$; platelet medians: $\mathrm{DENV}+=134896$ cells $/ \mathrm{ml}$ and DENV-= 223872 cells $/ \mathrm{ml}$ ) Transformed logarithmic values are presented in Table 1 . Percentages of atypical lymphocytes were lower among negative patients (medians: DENV $+=4 \%$ and DENV- $=1 \%$ ). In both groups, hematological variables were mutually strongly correlated. Whereas in leukocyte and platelet counts this was positive, in atypical lymphocytes it was negative (Table 2). With the exception of DAY and platelet counts in DENV-patients, there were no correlations between the variables SEX, DAY and AGE or any other variable in any group.

Logistic regression showed significant LEU and PLA usefulness in foreseeing DENV serological outcome, as reinforced by models with individually presented factors
Table 2 Spearman rank correlation among demographic and hematological variables for DENV+ and DENV- patients

\begin{tabular}{lllll}
\hline & DENV+ & & DENV- \\
$X \times Y$ & Rs & $P$ & Rs & $P$ \\
\hline SEX $\times$ DAY & -0.023 & 0.6847 & -0.078 & 0.3696 \\
SEX $\times$ AGE & -0.093 & 0.0971 & -0.017 & 0.8421 \\
SEX $\times$ LEU & 0.012 & 0.8287 & -0.142 & 0.1020 \\
SEX $\times$ PLA & -0.083 & 0.1379 & -0.185 & 0.0326 \\
SEX $\times$ AL\% & 0.026 & 0.6422 & 0.112 & 0.1985 \\
DAY $\times$ AGE & 0.733 & 0.0191 & 0.202 & 0.1110 \\
DAY $\times$ LEU & -0.016 & 0.7739 & -0.171 & 0.0488 \\
DAY $\times$ PLA & 0.062 & 0.2679 & -0.299 & $\mathbf{0 . 0 0 0 4}$ \\
DAY $\times$ AL\% & 0.139 & 0.0124 & 0.104 & 0.2303 \\
AGE $\times$ LEU & 0.094 & 0.0915 & -0.061 & 0.4872 \\
AGE $\times$ PLA & 0.030 & 0.5974 & -0.172 & 0.0475 \\
AGE $\times$ AL\% & -0.038 & 0.4954 & 0.045 & 0.6053 \\
LEU $\times$ PLA & 0.496 & $<\mathbf{0 . 0 0 0 1}$ & 0.336 & $\mathbf{0 . 0 0 0 1}$ \\
LEU $\times$ AL\% & -0.318 & $<\mathbf{0 . 0 0 0 1}$ & -0.376 & $<\mathbf{0 . 0 0 0 1}$ \\
PLA $\times$ AL\% & -0.482 & $<\mathbf{0 . 0 0 0 1}$ & -0.274 & $\mathbf{0 . 0 0 1 4}$ \\
\hline SPe & & &
\end{tabular}

Spearman rank correlation ( $R_{S}$, and their respective $P$-values) among demographic and hematological variables in two groups of patients (DENV $+N=322$, DENV- $N=134$ ). Figures lower than critical $P$ were in bold. For 15 correlation tests in each group of patients, critical $P=0.05 / 15=0.0033$. Variable codes as in Table 1

for the entire dataset (Table 3). As already mentioned, these two variables were strongly correlated in both groups (DENV+ and DENV-; Table 2). The contribution of each factor (except SEX) as DENV-infection predictors was presented in sigmoidal curves (Fig. 1). When using the entire dataset, SEX, DAY and AGE proved to be non-significant factors (in the latter two, only proven after Bonferroni correction for critical $P$ ) in their respective and exclusive models. Among all the subsets, LEU and PLA alone remained individually significant factors. AL\% (s1 and s4) and DAY (s3) also appeared punctually in the subsets as significant individual factors.

In the all-inclusive additive logistic regression model for evaluating the contribution of the six DENVinfection explanatory factors (Table 4), only LEU and PLA presented significant effects in the entire dataset. LEU presented significant results in all the subsets (only this in s1, and with DAY in s3 and AL\% in s4), except in s2. In this, and as AGE and PLA had been discarded by Bonferroni correction, no factor could be considered significant. In no subset did SEX, AGE and PLA appear as significant factors. A model using only LEU and PLA additively for the entire dataset resulted in an AIC value (409.4) lower than those of both "individualized" models (423.6 for LEU and 457.2 for PLA; Table 3). This twofactor model was slightly better than the "full" additive 
Table 3 Logistic regression models of demographic and hematological factors as individual predictors of DENV infection

\begin{tabular}{|c|c|c|c|c|c|c|}
\hline \multirow[b]{2}{*}{ Factors } & \multirow[b]{2}{*}{ Statistics } & \multicolumn{5}{|l|}{ Dataset } \\
\hline & & All & s1 & s2 & s3 & s4 \\
\hline \multirow[t]{7}{*}{ Sex } & $\beta_{0}$ & 0.774 & 0.392 & 1.276 & 0.928 & 0.649 \\
\hline & $\beta_{1}$ & 0.226 & 0.160 & -0.288 & 0.325 & 0.660 \\
\hline & OR & 1.254 & 1.173 & 0.750 & 1.384 & 1.934 \\
\hline & $C L-$ & 0.8352 & 0.5494 & 0.3175 & 0.5899 & 0.8172 \\
\hline & $\mathrm{CL}+$ & 1.8823 & 2.5068 & 1.7716 & 3.2458 & 4.5777 \\
\hline & $P$ & 0.2752 & 0.6794 & 0.5119 & 0.4553 & 0.1335 \\
\hline & AIC & 551.1 & 155.9 & 130.7 & 132.7 & 138.9 \\
\hline \multirow[t]{7}{*}{ Day } & $\beta_{\circ}$ & 0.435 & 0.312 & 2.048 & -7.409 & -1.499 \\
\hline & $\beta_{1}$ & 0.011 & 0.010 & -0.027 & 0.178 & 0.036 \\
\hline & OR & 1.011 & 1.010 & 0.973 & 1.194 & 1.037 \\
\hline & $C L-$ & 1.0009 & 0.9635 & 0.8820 & 1.0682 & 0.9876 \\
\hline & $\mathrm{CL}+$ & 1.0214 & 1.0593 & 1.0737 & 1.3350 & 1.0891 \\
\hline & P & 0.0334 & 0.6728 & 0.5868 & 0.0018 & 0.1443 \\
\hline & $\mathrm{AIC}$ & 547.7 & 155.9 & 130.8 & 122.2 & 138.9 \\
\hline \multirow[t]{7}{*}{ Age } & $\beta_{\circ}$ & 0.427 & 0.033 & 0.292 & 0.615 & 0.879 \\
\hline & $\beta_{1}$ & 0.013 & 0.013 & 0.025 & 0.013 & 0.001 \\
\hline & OR & 1.013 & 1.013 & 1.025 & 1.014 & 1.001 \\
\hline & $C L-$ & 1.0020 & 0.9934 & 0.9999 & 0.9896 & 0.9789 \\
\hline & $\mathrm{CL}+$ & 1.0242 & 1.0331 & 1.0503 & 1.0379 & 1.0226 \\
\hline & $P$ & 0.0210 & 0.1948 & 0.0510 & 0.2717 & 0.9628 \\
\hline & AIC & 546.8 & 154.3 & 126.9 & 132.1 & 141.2 \\
\hline \multirow[t]{7}{*}{ LEU } & $\beta_{\circ}$ & 24.173 & 32.162 & 18.422 & 20.852 & 25.583 \\
\hline & $\beta_{1}$ & -6.3036 & -8.4818 & -4.6950 & -5.3871 & -6.7062 \\
\hline & OR & 0.002 & 0.001 & 0.009 & 0.005 & 0.001 \\
\hline & $C L-$ & 0.0005 & 0.0000 & 0.0009 & 0.0004 & 0.0001 \\
\hline & $\mathrm{CL}+$ & 0.0069 & 0.0048 & 0.0972 & 0.0594 & 0.0206 \\
\hline & P & $<0.0001$ & $<0.0001$ & $<0.0001$ & $<0.0001$ & $<0.0001$ \\
\hline & $\mathrm{AlC}$ & 440.6 & 99.9 & 111.3 & 110.7 & 107.4 \\
\hline \multirow[t]{7}{*}{ PLA } & $\beta_{\circ}$ & 31.916 & 43.426 & 34.360 & 30.579 & 19.594 \\
\hline & $\beta_{1}$ & -5.931 & -8.143 & -6.377 & -5.646 & -3.586 \\
\hline & OR & 0.003 & 0.001 & 0.002 & 0.003 & 0.028 \\
\hline & $C L-$ & 0.0007 & 0.0001 & 0.0001 & 0.0002 & 0.0027 \\
\hline & $\mathrm{CL}+$ & 0.0108 & 0.0068 & 0.0359 & 0.0705 & 0.2855 \\
\hline & P & $<0.0001$ & $<0.0001$ & $<0.0001$ & 0.0002 & 0.0026 \\
\hline & AlC & 464.6 & 109.8 & 106.7 & 115.1 & 130.3 \\
\hline \multirow[t]{4}{*}{ AL\% } & $\beta_{\circ}$ & 0.192 & -0.287 & 0.547 & 0.672 & -0.242 \\
\hline & $\beta_{1}$ & 0.169 & 0.209 & 0.207 & 0.077 & 0.251 \\
\hline & OR & 1.184 & 1.233 & 1.230 & 1.080 & 1.285 \\
\hline & CL- & 1.1190 & 1.0950 & 1.0480 & 0.9891 & 1.1274 \\
\hline
\end{tabular}

Table 3 Logistic regression models of demographic and hematological factors as individual predictors of DENV infection (Continued)

\begin{tabular}{llllll}
\hline $\mathrm{CL}+$ & 1.2538 & 1.3875 & 1.4439 & 1.1793 & 1.4654 \\
$\mathrm{P}$ & $<\mathbf{0 . 0 0 0 1}$ & $\mathbf{0 . 0 0 0 5}$ & 0.0113 & 0.0860 & $\mathbf{0 . 0 0 0 2}$ \\
$\mathrm{AIC}$ & 511.8 & 136.1 & 118.9 & 129.8 & 118.1
\end{tabular}

Logistic regression models to evaluate the individual contribution of demographic and hematological factors to DENV incidence using different datasets ( $A L L=$ entire data; subsets $s 1$ to $s 4$ ordered by day of the first attendance at the Marilia Hemocenter; see text). $\beta_{\mathrm{o}}=$ intercept, $\beta_{1}=$ slope, $O R$ odds ratio, and $C L 95 \%$ confidence limits. Figures lower than critical $P$ were in bold. Considering six models (one for each factor) with each dataset, critical $P=0.05 / 6=0.0083$. AIC indicate model fit (comparable both between subsets and factors for subsets s1 to s4; comparable between factors for ALL). Variable codes as in Table 1

model $(\mathrm{AIC}=412.1)$ with all the six explanatory variables (Table 4).

According to both sensitivity and specificity, the factors AGE and DAY differed slightly from chance in DENV discrimination: their ROC curves appear near the graph diagonal (Fig. 2, Table 5). LEU and PLA, in this order, were considered the best factors by simultaneously reaching the highest scores of true positives and true negatives, as indicated by high AUC values (Fig. 2, Table 5). The AL\% was placed intermediately between the two groups of variables (Fig. 2, Table 5). Except for s3 in DAY, ROC curves computed for each factor of the subsets were not statistically different from those compiled for the entire dataset (Table 6).

\section{Discussion}

Clinical criteria for post-epidemiological threshold phase DF diagnosis have been largely employed in developing countries, due to the technical and economic difficulties in carrying out specific tests to confirm DENV infection during outbreaks [23]. In these cases, blood-cell counts of patients with DF symptoms are the procedure in healthcare institutions, as a way of monitoring disease evolution with the purpose of preventing and treating the severe hemorrhagic form [24, 25]. Potentially, clinical signs and the hematological profile of patients with acute DENV infection can vary individually and regionally, due to epidemiological characteristics, such as the presence of other seasonal infectious diseases, host and virus genetic background, and previous virus circulation, thereby making the use of only clinical criteria for diagnosis questionable [26]. In order to investigate the effectiveness of such criteria applied to febrile patients at the Marilia Hemocenter during the 2007 outbreak, demographic and hematological status were assessed by applying a posteriori NS1-based DENV serological outcome data.

Through presenting high sensitivity and specificity in studies carried out in Brazil, NS1 antigen detection has 

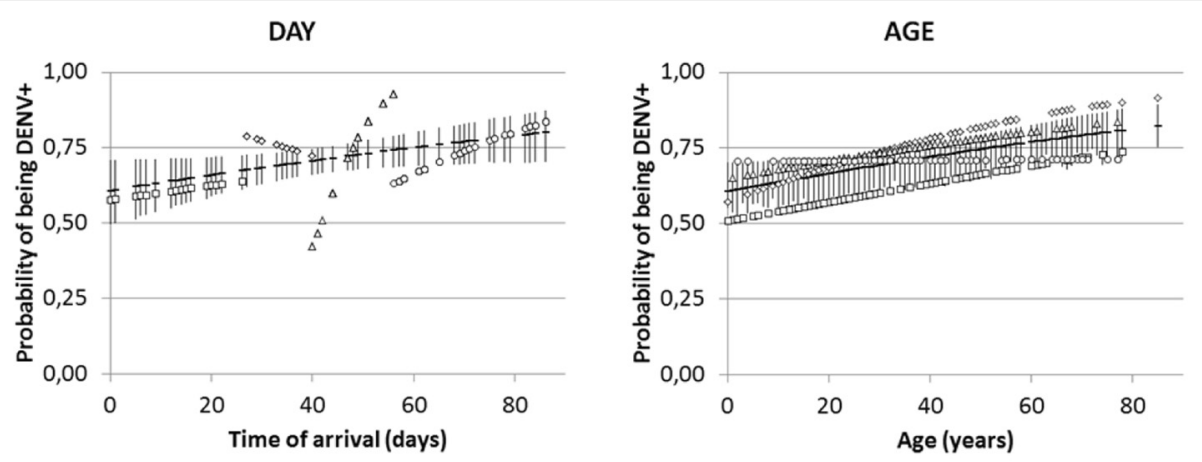

LEU
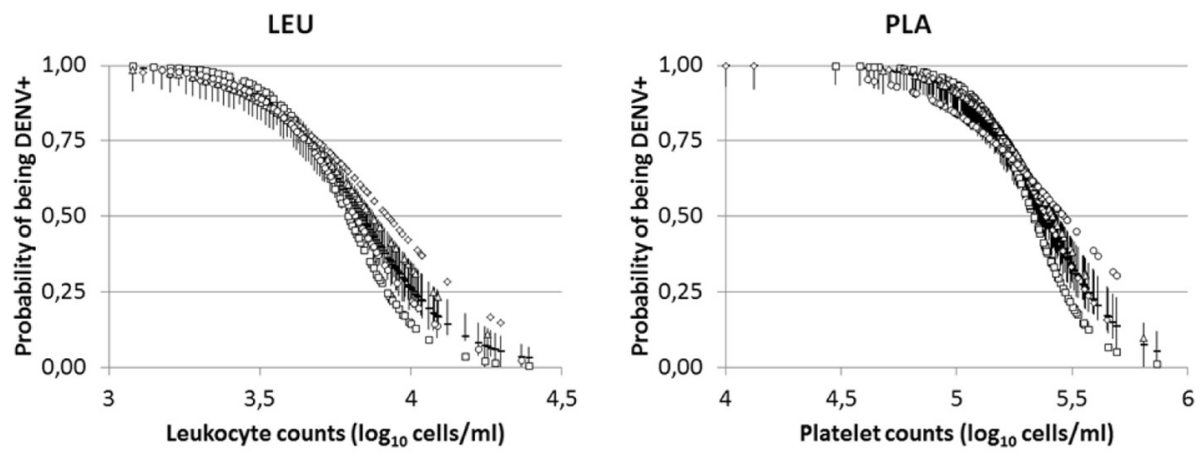

$\mathrm{AL} \%$

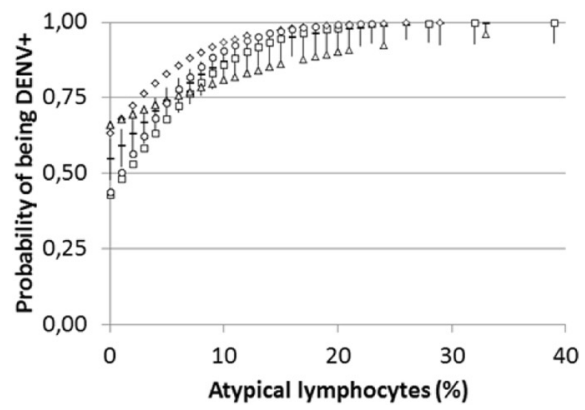

Fig. 1 Sigmoidal curves showing individual contribution of demographic and hematological factors as independent predictors of DENV infection, based on parameters of logistic regression in Table 2. Horizontal bars represent the entire dataset (with associated $95 \%$ confidence intervals). Subsets (see text) are $s 1=$ squares, $s 2=$ diamonds, s3 = triangles, s4 = circles. Variable codes as in Table 1

proved to be an important tool in early DF diagnosis [27-30]. The kit employed in this investigation has an expected Positive Percent Agreement (PPA) of $88.2 \%$ (95 \% Confidence Interval: 73.4-95.3\%) and a Negative Percent Agreement (NPA) of 100 \% (95\%CI: 93.2$100.0 \%$ ) (manufacturer's information). Accordingly, clinical criteria success was confirmed in $70.6 \%$ of the cases reported. The remainder (29.4\%) was comprised of samples with a false DENV outcome or which corresponded to other infectious diseases. In order to increase the accuracy of DENV NS1 serological diagnosis, the detection of IgM/IgG DENV NS1 antibodies is recommended $[31,32]$. However it was impossible to do so in the present study. Furthermore, serological tests to check the presence of the different infectious agents were omitted here.
Even though indicator efficiency of hematological changes during progression in distinguishing DENV from other infectious diseases is controversial, it remains essential in the clinical management of DF and severe hemorrhagic dengue $[25,33]$. Leukocyte and platelet counts below $3760 \mathrm{cell} / \mathrm{ml}$ and $100000 \mathrm{cells} / \mathrm{ml}$, respectively, and the presence of atypical lymphocytes, are commonly found in classical and severe cases of DF $[20,34,35]$. Our data showed a strongly significant association of decreased leukocyte and platelet counts in DENV+ patients. ROC curves reinforced LEU and PLA as the best factors for predicting serological DENV outcomes (Fig. 1).

Biochemical liver damage markers, such as aspartate aminotransferase (AST), and serum glutamic oxaloacetic transaminase (SGOT), as well as alanine aminotransferase 
Table 4 All inclusive, additive logistic regression models of demographic and hematological factors as predictors of DENV infection

\begin{tabular}{|c|c|c|c|c|c|c|}
\hline & & Dataset & & & & \\
\hline & Statistics & ALL & s1 & s2 & s3 & s4 \\
\hline & $\mathrm{AIC}$ & 412.1 & 97.5 & 107.1 & 103.4 & 98.1 \\
\hline Factors & $\beta_{\circ}$ & 30.194 & 54.258 & 30.300 & 31.291 & 15.871 \\
\hline \multirow[t]{5}{*}{ Sex } & $\beta_{1}$ & -0.007 & -1.006 & 0.1092 & 0.3120 & 0.7480 \\
\hline & OR & 0.993 & 0.366 & 1.115 & 1.366 & 2.113 \\
\hline & CL- & 0.6035 & 0.1079 & 0.3908 & 0.4545 & 0.6700 \\
\hline & $\mathrm{CL}+$ & 1.6342 & 1.2385 & 3.1827 & 4.1066 & 6.6626 \\
\hline & $P$ & 0.9784 & 0.1060 & 0.8383 & 0.5785 & 0.2018 \\
\hline \multirow[t]{5}{*}{ Day } & $\beta_{1}$ & 0.002 & -0.050 & -0.007 & 0.197 & 0.073 \\
\hline & OR & 1.002 & 0.951 & 0.993 & 1.218 & 1.076 \\
\hline & CL- & 0.9900 & 0.8872 & 0.8854 & 1.0630 & 1.0049 \\
\hline & $\mathrm{CL}+$ & 1.0145 & 1.0197 & 1.1132 & 1.3953 & 1.1520 \\
\hline & $P$ & 0.7310 & 0.1584 & 0.9012 & 0.0045 & 0.0356 \\
\hline \multirow[t]{5}{*}{ Age } & $\beta_{1}$ & 0.011 & 0.006 & 0.035 & 0.018 & -0.028 \\
\hline & OR & 1.011 & 1.006 & 1.036 & 1.018 & 0.973 \\
\hline & CL- & 0.9985 & 0.9786 & 1.0055 & 0.9887 & 0.9426 \\
\hline & $\mathrm{CL}+$ & 1.0245 & 1.0339 & 1.0666 & 1.0488 & 1.0038 \\
\hline & $P$ & 0.0831 & 0.6755 & 0.0202 & 0.2274 & 0.0847 \\
\hline \multirow[t]{5}{*}{ LEU } & $\beta_{1}$ & -4.518 & -6.303 & -2.310 & -4.632 & -6.838 \\
\hline & OR & 0.011 & 0.002 & 0.099 & 0.010 & 0.001 \\
\hline & CL- & 0.0025 & 0.0000 & 0.0049 & 0.0004 & 0.0000 \\
\hline & $\mathrm{CL}+$ & 0.0477 & 0.0840 & 20.160 & 0.2510 & 0.0430 \\
\hline & $P$ & $<0.0001$ & 0.0012 & 0.1327 & 0.0052 & 0.0003 \\
\hline \multirow[t]{5}{*}{ PLA } & $\beta_{1}$ & -2.536 & -5.556 & -4.194 & -4.392 & 0.947 \\
\hline & OR & 0.079 & 0.004 & 0.015 & 0.012 & 2.578 \\
\hline & CL- & 0.0150 & 0.0000 & 0.0003 & 0.0003 & 0.1099 \\
\hline & $\mathrm{CL}+$ & 0.4192 & 0.3552 & 0.7826 & 0.5545 & 60.4591 \\
\hline & $P$ & 0.0029 & 0.0160 & 0.0374 & 0.0236 & 0.5564 \\
\hline \multirow[t]{5}{*}{ AL\% } & $\beta_{1}$ & 0.040 & 0.034 & 0.073 & -0.090 & 0.262 \\
\hline & OR & 1.041 & 1.035 & 1.076 & 0.914 & 1.299 \\
\hline & CL- & 0.9828 & 0.9227 & 0.9101 & 0.8157 & 1.104 \\
\hline & $\mathrm{CL}+$ & 1.1029 & 1.1603 & 1.2724 & 1.0245 & 1.529 \\
\hline & $P$ & 0.1703 & 0.5592 & 0.3909 & 0.1227 & 0.0017 \\
\hline
\end{tabular}

All-inclusive, additive logistic regression models to evaluate the contribution of demographic and hematological factors to DENV incidence using different datasets ( $A L L=$ entire data; subsets $s 1$ to $s 4$ ordered by day of the first attendance at the Marilia Hemocenter; see text). AIC indicate model fit (comparable only between subsets $s 1$ to $s 4$ ). $\beta_{\mathrm{o}}=$ intercept, $\beta_{1}=$ slope, $\mathrm{OR}=$ odds ratio, and $\mathrm{CL}=95 \%$ confidence limits. Figures lower than critical $\mathrm{P}$ were in bold. Considering five models (one for each dataset), critical $P=0.05 / 5=0.01$. Variable codes as in Table 1

(ALT) and serum glutamic pyruvic transaminase (SGPT) serum levels, are used to evaluate dengue disease severity [2]. However, in our health system these parameters are absent from the normal hematological exam routine, and thus could not be included in the study.

Data of the demographic status of a population involved in a DENV outbreak facilitates characterization of the epidemiological pattern of virus transmission, thereby contributing towards prevention and management. In Asia, where the number of severe hemorrhagic dengue cases is the highest in the world, with a fatality rate of $3.5 \%$, prevalence is higher in children under15 [36]. In the 2007 Marilia DENV outbreak, the adult population was mostly affected by DF, with only five cases of the non-lethal hemorrhagic form of the disease $(0.4 \%)$. No differences in gender or age were found among DENV+ and DENV- patients. This DENV transmission pattern is the most common in the Americas [9].

In the different regions of Brazil, the time span of DF outbreaks after the establishment of an epidemiological threshold, is long-lasting, and can continue throughout the hot rainy season. In São Paulo State it can last for four to six months [37]. During this period, health care facilities are overcrowded and there is confusion in diagnosis with seasonal diseases. Moreover, and depending on the course of infection, it is possible that hematologic changes, so commonly associated with the more severe DENV diseases are not very evident in initial data. Thus, in order to further adapt monitoring of the disease, all patients suspected of DENV infection are assayed for hematological changes [38]. By taking into consideration the regional demographic and hematological profiles of DF and its more severe hemorrhagic form [39, 40], the early definition of these characteristics can improve management efficiency. Thus, in order to guarantee maintenance of parameters throughout the 2007 Marilia outbreak, the patients were divided into four temporally consecutive subsets for comparative analysis. The results indicated temporal homogeneity in demographic and hematological profiles, especially brought into evidence by logistic regression and ROC curves (Table 3 and Fig. 2). This showed LEU and PLA | to be independent factors associated to DENV serological positive diagnosis during the whole period.

There was also a statistically significant association of hematological variables in all DENV serological positive samples (Table 5), compatible with DENV infection laboratory findings [37]. However, certain differences were found by additive logistic regression analysis, when comparing the entire dataset and subsets s1 to s4 (Tables 4 and 6). The most significant difference occurred in subset s3, which presented high correlations with DENV positive serological diagnosis. This difference could be attributed to specific unidentified features related to sampling during the period, since this was the only 


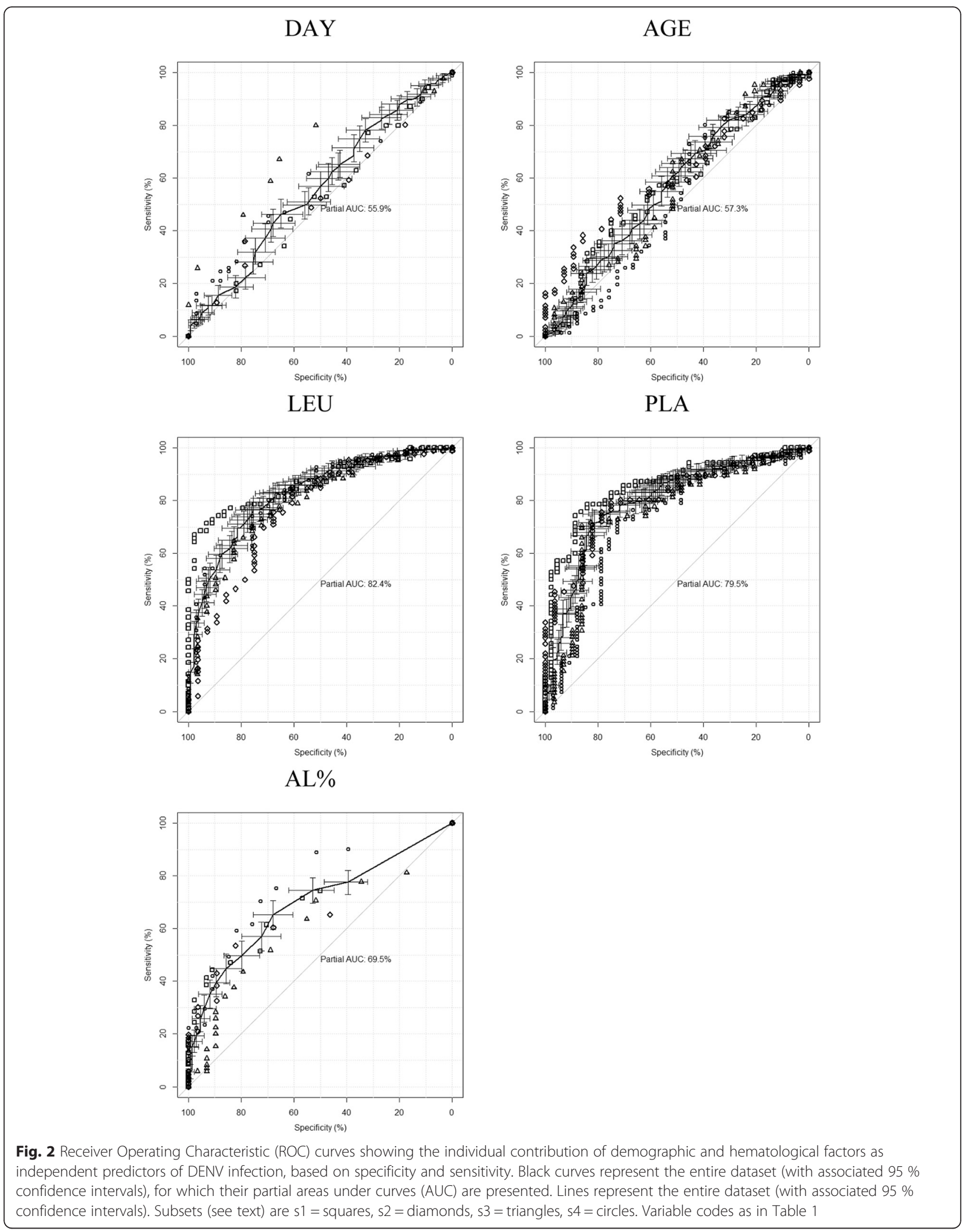


Table 5 Paired comparison among Receiver Operating

Characteristic (ROC) curves among demographic and hematological factors as predictors of DENV infection

\begin{tabular}{|c|c|c|c|c|c|}
\hline Factor A & Factor B & $A \cup C_{A}$ & $A \cup C_{B}$ & $\mathrm{D}$ & $P$ \\
\hline LEU & PLA & $82.41(77.08-85.88)$ & 79.54 (71.72-81.84) & 1.88 & 0.0595 \\
\hline LEU & AL\% & & $69.51(65.63-77.56)$ & 3.07 & 0.0021 \\
\hline LEU & AGE & & $57.32(51.47-62.99)$ & 6.77 & $<0.0001$ \\
\hline LEU & DAY & & $55.86(51.21-62.45)$ & 7.28 & $<0.0001$ \\
\hline PLA & AL\% & & & 1.45 & 0.1462 \\
\hline PLA & AGE & & & 5.52 & $<0.0001$ \\
\hline PLA & DAY & & & 5.56 & $<0.0001$ \\
\hline AL\% & AGE & & & 3.57 & 0.0004 \\
\hline AL\% & DAY & & & 3.80 & 0.0001 \\
\hline AGE & DAY & & & 0.03 & 0.9733 \\
\hline
\end{tabular}

AUC is area under the curve (\%) and D is the statistics related to the difference between curves. $P$ values lower than critical were in bold. Considering ten contrasts, critical $P=0.05 / 10=0.005$. Factors appear in decreasing order of AUC, showed only at first time with respective $95 \%$ confidence limits in parenthesis. Variable codes as in Table 1

statistical significant variable to be associated to DENV diagnosis.

Currently, methods for the specific treatment and effective prevention of DENV infection are still unavailable. Furthermore, a specific and sensitive method for

Table 6 Paired comparison among Receiver Operating Characteristic (ROC) curves between subsets and the entire data for demographic and hematological factors as predictors of DENV infection

\begin{tabular}{|c|c|c|c|c|c|c|}
\hline & & Dataset & & & & \\
\hline Factor & Statistics & ALL & s1 & s2 & s3 & s4 \\
\hline \multirow[t]{3}{*}{ Day } & AUC & 55.86 & 51.61 & 52.49 & 70.24 & 57.95 \\
\hline & $D$ & & 0.65 & 0.52 & -2.41 & -0.32 \\
\hline & P & & 0.5148 & 0.5970 & 0.0160 & 0.7449 \\
\hline \multirow[t]{3}{*}{ Age } & AUC & 57.32 & 57.60 & 61.57 & 56.47 & 52.19 \\
\hline & $D$ & & -0.04 & -0.67 & 0.12 & 0.69 \\
\hline & P & & 0.9655 & 0.5043 & 0.9040 & 0.4873 \\
\hline \multirow[t]{3}{*}{ LEU } & AUC & 82.41 & 86.82 & 76.26 & 78.97 & 82.25 \\
\hline & D & & -1.16 & 1.06 & 0.67 & 0.03 \\
\hline & P & & 0.2474 & 0.2894 & 0.5041 & 0.9726 \\
\hline \multirow[t]{3}{*}{ PLA } & AUC & 79.54 & 85.57 & 80.67 & 75.80 & 72.45 \\
\hline & $D$ & & -1.38 & -0.23 & 0.64 & 1.21 \\
\hline & $P$ & & 0.1687 & 0.8152 & 0.5209 & 0.2269 \\
\hline \multirow[t]{3}{*}{$\mathrm{AL} \%$} & AUC & 69.51 & 70.67 & 66.34 & 61.87 & 77.61 \\
\hline & D & & -0.22 & 0.59 & 1.18 & -1.55 \\
\hline & P & & 0.8234 & 0.5574 & 0.2365 & 0.1222 \\
\hline
\end{tabular}

AUC is area under the curve (\%) and D is the statistics related to the difference between curves. All $P$ values are above the critical (critical $P=0.05 / 4=0.0125$, considering four contrasts for each factor). The only significant figure before such correction is in bold. Variable codes as in Table 1 early DENV-diagnosis is not accessible to all the regions and patients during the course of long dengue epidemics in developing countries, this including Brazil. In order to improve dengue management in this situation, an alternative strategy used by public health care institutions in São Paulo State is, according to the size of the population, determine an epidemiological threshold after the epidemic has reached the incidence rate. Before this, patients undergo dengue laboratory diagnosis. Considering our results, if the epidemiological and hematological profile patterns of the dengue-affected part of the population are investigated in the beginning of the outbreak, when specific diagnosis is carried out after the threshold is reached, the resultant hematological profiles could be used with more accuracy to improve disease management. Furthermore, to reduce the number of dengue false-positives from exclusive clinical-criteria diagnosis, individuals notified as being infected but presenting high scores for LEU and PLA, could be submitted to dengue serological testing. Thus, definition of the epidemiological pattern of DENV transmission in regions with recurrent outbreaks could help healthcare institutions to prevent virus spread and manage associated diseases.

\section{Conclusions}

Herein it was shown that at the outbreak, in a middlesized town of São Paulo State, Brazil, the clinical criteria applied after an epidemiological threshold has been reached was efficient in managing both classical and more severe cases of DF, since more than $70 \%$ of the reported cases had been confirmed by serologic detection of the DENV NS1 antigen, associated to low leukocyte and platelet counts. The epidemiological pattern of DENV transmission in the town of Marilia was similar to the American prototype, with a prevalence of DF in the adult population and the low occurrence of hemorrhagic forms $(0.4 \%)$. Furthermore, demographic and hematological profiles showed a conservative temporal pattern which points to the possibility of making use of the characteristics of an outbreak detected early in the beginning, as a means of improving management throughout all the remaining period. Furthermore, public healthcare institutions could be instructed on how to inform any change in the specific pattern of the outbreak, and thus facilitate investigation of the possible simultaneous occurrence of a new disease, as well the opportune local entry of a different circulating DENV genotype.

\section{Additional file}

Additional file 1: Multilingual abstracts in the five official working languages of the United Nations. (PDF $386 \mathrm{~kb}$ ) 


\section{Abbreviations}

AGE, age; AlC, akaike information criteria; AL\%, percentage of atypical lymphocytes; ALT, alanine aminotransferase; AST, aspartate aminotransferase; C, capsid; DENV, denque virus; DF, dengue fever; DHF, dengue hemohrragic fever; DSS, dengue shock syndrome; E, envelope; LEU, leukocyte; NS, nonstructural protein; PLA, platelets; prM, pre-membrane; ROC, receiver operating characteristic; SEX, gender; SGOT, serum oxaloacetic transaminase; SGPT, serum glutamic pyruvic transaminase

\section{Acknowledgement}

This study received financial support from PRONEX/Dengue, CNPq, Grant number 550106/2010-3. All authors are grateful to Dr. Ramon Arthus Clark which contributed to improve the final version of the article.

\section{Authors' contributions}

AMSC carried out the serologic studies, participated in the design of the study, as well as hematological profile determination, and helped in drafting the manuscript; RBS participated in the design of the study and demographic and hematological data analysis; MMR participated in the serological study and hematological data analysis; AE participated in the design of the study and drafting of the manuscript, as well as all statistical analysis; MAS conceived the study, participated in its design and coordination, and helped to draft the manuscript. All the authors read and approved the final manuscript.

\section{Competing interests}

All the authors of this paper declare that in the past three years, they have not received reimbursements, fees, funding, or salaries from any organization that may in any way gain or lose financially from the publication of this manuscript, now or in the future. All the authors also declare that any stock or shares in any organization that may in any way gain or lose financially from the publication of this manuscript are not now in their possession, neither will be in the future. Furthermore, all the authors declare that currently no patents relating to the content of the manuscript are being applied for, nor have they received reimbursements, fees, funding, or salaries from any organization that holds, or has applied for, patents relating to the contents of the manuscript. All the authors also declare that there are no other financial or non-financial competing interests.

\section{Author details}

${ }^{1}$ Center for Natural and Human Sciences, Universidade Federal do ABC, Campus São Bernardo do Campo, 09606-070 São Bernardo Do Campo, São Paulo, Brasil. ${ }^{2}$ Secretaria do Estado da Saúde do Estado de São Paulo, Instituto Adolfo Lutz, Centro de Laboratório Regional VIII, Santo André 09040-160, São Paulo, Brazil. ${ }^{3}$ Discipline of Parasitology, Marilia Medical School, Marilia 17519-030, São Paulo, Brazil. ${ }^{4}$ Center for Natural and Human Sciences, Universidade Federal do ABC, Campus Santo André, Santo André 09210-170, São Paulo, Brazil.

\section{Received: 15 September 2015 Accepted: 25 July 2016 Published online: 05 September 2016}

\section{References}

1. Lambrechts L, Scott TW, Gubler DJ. Consequences of the expanding global distribution of Aedes albopictus for dengue virus transmission. Plos Neglectet Trop Dis. 2010. doi:10.1371/journal.pntd.0000646.

2. Dengue. Guidelines for diagnosis, treatment, prevention and control: new edition. Geneva: WHO Guidelines Approved by the Guidelines Review Committee; 2009.

3. Tang KF, Ooi EE. Diagnosis of dengue: an update. Expert Rev Anti Infect Ther. 2012. doi:10.1586/eri.12.76.

4. Gubler DJ. Dengue and dengue hemorrhagic fever. Clin Microbiol Rev. 1998;11:480-96.

5. Halstead SB. Dengue in the Americas and Southeast Asia: do they differ? Rev Panam Salud publica/Pan Am J Public Health. 2006;20:407-15.

6. Cordeiro R, Donalisio MR, Andrade VR, Mafra AC, Nucci LB, Brown JC, et al. Spatial distribution of the risk of dengue fever in southeast Brazil, 20062007. BMC Public Health. 2011. doi:10.1186/1471-2458-11-355.

7. Brady OJ, Smith DL, Scott TW, Hay SI. Dengue disease outbreak definitions are implicitly variable. Epidemics. 2015. doi:10.1016/j.epidem.2015.03.002.

8. San Martin JL, Brathwaite O, Zambrano B, Solorzano JO, Bouckenooghe A, Dayan $\mathrm{GH}$, et al. The epidemiology of dengue in the americas over the last three decades: a worrisome reality. Am J Trop Med Hyg. 2010. doi:10.4269/ ajtmh.2010.09-0346.

9. Brathwaite Dick O, San Martin JL, Montoya RH, del Diego J, Zambrano B, Dayan GH. The history of dengue outbreaks in the Americas. Am J Trop Med Hyg. 2012. doi:10.4269/ajtmh.2012.11-0770.

10. Teixeira MG, Costa Mda C, Barreto F, Barreto ML. Dengue: twenty-five years since reemergence in Brazil. Cad Saude Publica. 2009;25 Suppl 1:S7-18.

11. Mondini A, Cardeal IL, Lazaro E, Nunes SH, Moreira CC, Rahal P, et al. Saint Louis encephalitis virus, Brazil. Emerg Infect Dis. 2007. doi:10.3201/eid1301.060856.

12. Albuquerque IG, Marandino R, Mendonca AP, Nogueira RM, Vasconcelos PF, Guerra $L R$, et al. Chikungunya virus infection: report of the first case diagnosed in Rio de Janeiro, Brazil. Rev Soc Bras Med Trop. 2012;45:128-9.

13. Donalisio MR, Freitas AR. Chikungunya in Brazil: an emerging challenge. Rev Bras Epidemiol. 2015. doi:10.1590/1980-5497201500010022.

14. Roth A, Mercier A, Lepers C, Hoy D, Duituturaga S, Benyon E, et al. Concurrent outbreaks of dengue, chikungunya and Zika virus infections - an unprecedented epidemic wave of mosquito-borne viruses in the Pacific 2012-2014. Euro Surveill. 2014;19:41.

15. de Souza Al, Nogueira JM, Pereira MM. Anti-Leptospira antibodies in patients in the State of Mato Grosso do Sul with clinical suspicion of dengue or viral hepatitis. Rev Soc Bras Med Trop. 2007:40:431-5.

16. Mishra B, Singhal L, Sethi S, Ratho RK. Leptospirosis coexistent with dengue fever: a diagnostic dilemma. J Global Infect Dis. 2013. doi:10.4103/0974777X.116878.

17. Imbert P, Algayres JP, Daly JP, Hovette P. Visceral leishmaniasis: a case in Tahiti at the end of dengue after a 2-year asymptomatic carriage]. Presse Med. 1993;22:689.

18. Magalhaes BM, Alexandre MA, Siqueira AM, Melo GC, Gimaque JB, Bastos MS, et al. Clinical profile of concurrent dengue fever and Plasmodium vivax malaria in the Brazilian Amazon: case series of 11 hospitalized patients. Am J Trop Med Hyg. 2012. doi:10.4269/ajtmh.2012.12-0210.

19. Cavalcanti LP, Coelho IC, Vilar DC, Holanda SG, Escossia KN, Souza-Santos R. Clinical and epidemiological characterization of dengue hemorrhagic fever cases in northeastern, Brazil. Rev Soc Bras Med Trop. 2010;43:355-8.

20. Daumas RP, Passos SR, Oliveira RV, Nogueira RM, Georg I, Marzochi KB, et al. Clinical and laboratory features that discriminate dengue from other febrile illnesses: a diagnostic accuracy study in Rio de Janeiro, Brazil. BMC Infect Dis. 2013. doi:10.1186/1471-2334-13-77.

21. CVE. Centro de Vigilância Epidemiológica da Secretaria de Estado de Saúde do Estado de São Paulo. Manejo clínico de paciente com suspeita de dengue. http://portal.saude.sp.gov.br/resources/cve-centro-de-vigilanciaepidemiologica/areas-de-vigilancia/doencas-de-transmissao-por-vetores-ezoonoses/doc/dengue/dengue15_guia_pratico.pdf. Accessed 28 July 2016.

22. Robin X, Turck N, Hainard A, Tiberti N, Lisacek F, Sanchez JC, et al. pROC: an open-source package for $\mathrm{R}$ and $\mathrm{S}+$ to analyze and compare ROC curves. BMC Bioinformatics. 2011. doi:10.1186/1471-2105-12-77.

23. Diaz FA, Martinez RA, Villar LA. Clinical criteria to diagnose dengue in its early stages. Biomedica. 2006;26:22-30.

24. Nunes-Araujo FR, Ferreira MS, Nishioka SD. Dengue fever in Brazilian adults and children: assessment of clinical findings and their validity for diagnosis. Ann Trop Med Parasitol. 2003:97:415-9. doi:10.1179/000349803235002263.

25. Potts JA, Rothman AL. Clinical and laboratory features that distinguish dengue from other febrile illnesses in endemic populations. Trop Med Int Health. 2008. doi:10.1111/j.1365-3156.2008.02151.x.

26. Chacon-Duque JC, Adhikari K, Avendano E, Campo O, Ramirez R, Rojas W, et al. African genetic ancestry is associated with a protective effect on Dengue severity in colombian populations. Infect Genet Evol. 2014. doi:10.1016/j.meegid.2014.07.003.

27. Ehelepola ND, Ariyaratne K, Buddhadasa WM, Ratnayake S, Wickramasinghe M. A study of the correlation between dengue and weather in Kandy City, Sri Lanka (2003-2012) and lessons learned. Infect Dis Poverty. 2015. doi:10.1186/s40249-015-0075-8.

28. Castro-Jorge LA, Machado PR, Favero CA, Borges MC, Passos LM, de Oliveira RM, et al. Clinical evaluation of the NS1 antigen-capture ELISA for early diagnosis of dengue virus infection in Brazil. J Med Virol. 2010. doi:10.1002/jmv.21814.

29. Lima Mda R, Nogueira RM, Bispo de Filippis AM, dos Santos FB. Comparison of two generations of the Panbio dengue NS1 capture enzyme-linked immunosorbent assay. Clin Vaccine Immunol. 2011; doi:10.1128/CVI.00024-11.

30. Ferraz FO, Bomfim MR, Totola AH, Avila TV, Cisalpino D, Pessanha JE, et al. Evaluation of laboratory tests for dengue diagnosis in clinical specimens 
from consecutive patients with suspected dengue in Belo Horizonte, Brazil. JJ Clin Virol. 2013. doi:10.1016/j.jcv.2013.06.015.

31. Blacksell SD, Jarman RG, Bailey MS, Tanganuchitcharnchai A, Jenjaroen $K$, Gibbons RV, et al. Evaluation of six commercial point-of-care tests for diagnosis of acute dengue infections: the need for combining NS1 antigen and IgM/lgG antibody detection to achieve acceptable levels of accuracy. Clin Vaccine Immunol. 2011. doi:10.1128/CVI.05285-11.

32. Arya SC, Agarwal N. Simultaneous testing for dengue virus NS1, lgM, lgG, and counting of platelets. J Microbiol Immunol Infect. 2013. doi:10.1016/j. jmii.2013.01.007.

33. Tanner L, Schreiber M, Low JG, Ong A, Tolfvenstam T, Lai YL, et al. Decision tree algorithms predict the diagnosis and outcome of dengue fever in the early phase of illness. PLoS Neglect Trop Dis. 2008. doi:10.1371/journal.pntd. 0000196.

34. Liu JW, Lee IK, Wang L, Chen RF, Yang KD. The usefulness of clinicalpractice-based laboratory data in facilitating the diagnosis of dengue illness. Biomed Res Int. 2013. doi:10.1155/2013/198797.

35. Chadwick D, Arch B, Wilder-Smith A, Paton N. Distinguishing dengue fever from other infections on the basis of simple clinical and laboratory features: application of logistic regression analysis. J Clin Virol. 2006. doi:10.1016/j.jcv. 2005.06.002.

36. Ooi EE, Gubler DJ. Dengue in Southeast Asia: epidemiological characteristics and strategic challenges in disease prevention. Cad Saude Publica. 2009;25 Suppl 1:S115-24.

37. CVE. Centro de Vigilância Epidemiológica da Secretaria de Estado de Saúde do Estado de São Paulo. http://www.saude.sp.gov.br/resources/ccd/materiais-decomunicacao/dengue-2015/edicao_rev.pdf. Accessed 28 July 2016.

38. Oliveira EC, Pontes ER, Cunha RV, Froes IB, Nascimento D. Hematological abnormalities in patients with dengue. Rev Soc Bras Med Trop. 2009;42:682-5.

39. Guilarde AO, Turchi MD, Siqueira Jr JB, Feres VC, Rocha B, Levi JE, et al. Dengue and dengue hemorrhagic fever among adults: clinical outcomes related to viremia, serotypes, and antibody response. J Infect Dis. 2008. doi:10.1086/528805

40. Pires Neto Rda J, de Sa SL, Pinho SC, Pucci FH, Teofilo CR, Evangelista PD, et al. Dengue infection in children and adolescents: clinical profile in a reference hospital in northeast Brazil. Rev Soc Bras Med Trop. 2013; doi:10.1590/0037-8682-1716-2013.

\section{Submit your next manuscript to BioMed Central and we will help you at every step:}

- We accept pre-submission inquiries

- Our selector tool helps you to find the most relevant journal

- We provide round the clock customer support

- Convenient online submission

- Thorough peer review

- Inclusion in PubMed and all major indexing services

- Maximum visibility for your research

Submit your manuscript at www.biomedcentral.com/submit

) Biomed Central 